\section{The pulmonary artery catheter}

Maribel Manikon MRCP,

Registrar in Intensive Care

Michael Grounds MD MRCP,

Reader in Intensive Care

Andrew Rhodes MRCP FRCA,

Consultant in Intensive Care

Department of Intensive Care, St George's Hospital, London

Clin Med JRCPL 2002;2:101-4

The flow-directed pulmonary artery catheter (PAC) has been in clinical use since its introduction by Swan and colleagues in $1970^{1}$. During that time it has become widely used in critical care units throughout the world and is now the most commonly utilised form of flow monitoring. The haemodynamic data obtained with a PAC are used to monitor and modify therapy in the critically ill ${ }^{2}$, particularly those with haemodynamic instability. The use of the PAC is associated with risks ${ }^{3,4}$ which are compounded by the nature and severity of the patient's condition. A number of reports have suggested that these complications may outweigh the benefits incurred with the use of this monitor and accompanying editorials have called for a ban on its use $^{4-8}$.

\section{Design of the pulmonary artery catheter}

The PAC is a flexible balloon-tipped flow-directed catheter which, when inserted via central venous access, can be guided into a branch of the pulmonary artery. The PAC is commonly a triple lumen catheter with a proximal port for measuring right atrial pressure, a distal port for measuring pulmonary artery pressure, and a thermistor which allows calculation of cardiac output by thermodilution.

\section{Directly measured variables}

\section{The pulmonary artery wedge pressure}

Traditionally, an estimate of the preload has been obtained with the PAC, utilising the pulmonary artery wedge (or occlusion) pressure (PAWP). This is obtained when a balloon is inflated and occludes a branch of the pulmonary artery. With the balloon inflated, it is assumed that the pressure measured at the distal end of the PAC is the same as the left atrial pressure (LAP) and therefore the left ventricular end diastolic pressure (LVEDP). From the LVEDP may be estimated the left ventricular end diastolic volume (LVEDV) which is the best indicator of preload. The PAWP is always measured at the end of expiration. At this point in the respiratory cycle there is the minimum of interference from intrathoracic pressures being superimposed on the variable (extra positive pressure in positive pressure ventilation or negative pressures in spontaneously breathing patients).

\section{Key Points}

The pulmonary artery catheter directly measures cardiac output, pulmonary artery pressures and the oxygen saturations of blood through the heart and in the pulmonary arteries

The mixed venous oxygen saturation enables an estimate of the oxygen supply/demand balance to be made

The use of the pulmonary artery catheter in critically ill patients leads to a better understanding of the cardiovascular state of the patient

Data obtained from the pulmonary artery catheter are often misunderstood

KEY WORDS: CPD, assessment, cardiac, interpretation, monitor, oxygen, pulmonary, pulmonary artery catheter, shock, Swan-Ganz
The theory that PAWP provides an estimate of LVEDP holds true provided that the following assumptions are correct:

1 There is a direct uninterrupted column of blood between the pulmonary artery distal to the inflated balloon all the way to the left atrium.

2 There is no pathology of the pulmonary vasculature that would prevent the PAWP being the same as the LAP.

3 There is no mitral valve abnormality.

4 There is a linear relationship between LVEDP and LVEDV, allowing a comparison to be made between these two variables.

Clearly, some of these assumptions will not hold true in many sick patients and therefore the PAWP may not always be an accurate marker of preload.

\section{Cardiac output}

The PAC measures cardiac output by either an intermittent or continuous thermodilution technique in the pulmonary artery. Provided that there is neither a left-to-right nor a right-to-left shunt, this flow in the pulmonary artery provides a good estimate of cardiac output. It is important to monitor cardiac output in sick patients as there may be little or no correlation between cardiac output and blood pressure: that is, patients who have a good blood pressure may have a poor flow and vice versa. The measurement of 'flow' is important as it is often the only variable that can be manipulated to ensure an adequate delivery of oxygen to the tissues. The cardiac output can be used together with the heart rate to obtain the stroke volume of the heart.

\section{The mixed venous oxygen saturation}

The saturation of haemoglobin with oxygen in the pulmonary artery is known as the mixed venous oxygen saturation $\left(\mathrm{SvO}_{2}\right)$ and can be measured continuously with modern PACs by a reflectance spectrophotometry technique. This 
variable reflects the balance between the amount of oxygen delivered to the tissues and how much is used. A low $\mathrm{SvO}_{2}$ will therefore reflect either a decrease in the delivery of oxygen to the body or an increased oxygen utilisation. In most acutely sick patients a low $\mathrm{SvO}_{2}$ is due to a decreased oxygen delivery, either secondary to a low cardiac output or to anaemia, or to a primary problem with oxygenation. The $\mathrm{SvO}_{2}$ therefore allows the adequacy of any given cardiac output to be assessed for an individual patient.

\section{Indications for insertion of a pulmonary artery catheter}

There are no absolute indications for the insertion of a PAC. The usual indications include the use of the catheter for purely diagnostic applications and to guide therapy.

\section{Diagnostic applications}

The purely diagnostic applications include the use of the monitor to:

- predict myocardial ischaemia in anaesthetised patients

- assess cardiac and pulmonary performance prior to consideration for transplantation programmes

- identify intrinsic cardiac defects by measuring the oxygen saturation of blood in the different heart chambers.

\section{As a guide to therapy}

The use of the PAC to guide therapy includes:

- conditions in which fluid balance is difficult to control and monitor (eg an anuric patient with high insensible losses)

- the shocked patient who is not responding to standard treatments

- $\quad$ aiding the appropriate use and guidance of vasoactive therapies.

\section{Complications of the pulmonary artery catheter}

There have been numerous reports of complications associated with the
Table 1. Complications associated with the pulmonary artery catheter (PAC).

\section{Complication}

Associated with insertion of the PAC

- minor arrhythmias

- sustained arrhythmias

- arterial puncture

- pneumothorax

When the PAC is in place:

- infection of insertion site

- catheter-related sepsis

- mural thrombus

- pulmonary infarction

- rupture of pulmonary artery

- death

$\mathrm{PAC}^{2-4,7}$. These complications fall into one of three groups and result from:

- the gaining of central venous access

- the passing of the catheter through the right ventricle

- the presence of the PAC in the pulmonary artery (Table 1).

The most common complication is a transient arrhythmia as the catheter passes through the tricuspid and pulmonary valves. This arrhythmia, which can occur in up to $50 \%$ of insertions, is usually self-limiting and causes no harm. The more serious complications are those of pulmonary infarction, rupture and sudden death. These are extremely rare (less than 1 in 1,000 insertions) and should not occur with appropriate use and care of the catheters.

\section{Data interpretation}

The data obtained from the PAC must be interpreted in combination with other
Incidence (\%)

48
uncommon
1
1

$0-22$
2
$28-61$
$0.1-7$
$<0.1$
$<0.1$

markers of the patient's condition. Normal values for each of the variables obtained are detailed in Table 2 . However, the absolute value of any of these variables is often meaningless and it is the trend in response to treatment that is more relevant. The aim of using the PAC is to obtain an accurate assessment of the cardiovascular performance of the patient. This necessitates understanding of the Frank Starling relationship, which compares preload to cardiac work or stroke volume. For an individual patient, the PAWP and cardiac index or stroke volume should be measured from the PAC. A fluid challenge can then be given to the patient and the variables measured again. The fluid should continue to be given until the PAWP is at a level at which further increases cause no further rise in stroke volume. At this point, the patient is at the top of the 'Starling' curve and will not benefit from further increases in preload. If the cardiac output or stroke volume is still inad-
Table 2. Normal values of cardiac pressures $(\mathrm{mmHg})$ obtained from a pulmonary artery catheter in a spontaneously breathing patient.

\begin{tabular}{lcc} 
& Mean & Range \\
\hline Right atrium & 4 & $3-6$ \\
Right ventricle: & & \\
$\quad$ systolic & 25 & $20-30$ \\
$\quad$ diastolic & 4 & $2-8$ \\
Pulmonary artery: & & \\
$\quad$ systolic & 25 & $20-30$ \\
$\quad$ diastolic & 10 & $5-15$ \\
$\quad$ mean & 15 & $10-20$ \\
Pulmonary artery wedge pressure & 10 & $5-14$
\end{tabular}


equate for the patient (as assessed by end organ perfusion, development of lactic acidosis or $\mathrm{SvO}_{2}$ ), vasoactive medications need to be commenced, for instance a positive inotrope if the contractility of the heart is poor or a vasodilator if the problem is excessive afterload. Absolute values for each of these variables will obviously be different depending on the clinical circumstances, but using this approach to compare preload against cardiac performance can enable targets to be set for individual patients' therapy.

Clinicians and nurses are generally poor at understanding and interpreting data obtained from a PAC and have limited knowledge of how to make use of the data in a clinical setting ${ }^{9-11}$. These deficits include:

- interpretation of the waveforms associated with the PAC

- an understanding of the relevance of each variable

- identification of arterial or venous blood gases.

It is possible that the associated worries of an increase in mortality for patients managed with a PAC may be related to this 'educational' problem.

\section{The pulmonary artery catheter controversy}

Despite the PAC being in widespread clinical use for over 30 years, there is little published work demonstrating an improvement in outcome. In recent years there have been several reports of patients actually coming to harm through the use of this tool ${ }^{5,12,13}$. Connors et $a l^{5}$ examined the outcomes, length of intensive care unit (ICU) stay and hospital costs for 5,735 critically ill patients, of whom 2,184 were treated with the use of a PAC. To adjust for severity of illness, the authors used a propensity scoring system to represent the relationship between multiple covariates and the use of the PAC. Each patient receiving a $\mathrm{PAC}$ was paired with a patient with a comparable diagnosis and propensity score in whom a PAC was not used. There were fewer survivors among the patients treated with a PAC; this group also spent a longer time in the ICU and cost more because of the hospital stay.

There are several explanations for the findings in the Connors study:

1 The PAC may actually harm patients.

2 The use of the PAC may simply reflect a more aggressive form of care for these patients, and this type of care may be detrimental.

3 The clinicians inserting the PAC may not understand how to use it correctly or how to interpret the data appropriately.

4 It is possible that the patients who received a PAC were recognised by their clinicians to be sick and/or not responding to conventional therapy, and that this 'variable' was not identified as part of the propensity score (ie the patients were not well matched at baseline).

Several randomised controlled trials (RCTs) are in progress to ascertain whether the PAC does cause harm to critically ill patients. Early results suggest that the previously reported increase in mortality with the PAC is false. Rhodes et $a^{14}$ studied 201 critically ill patients with or without the use of a PAC and found that, while it conferred no appreciable benefit, the PAC did not increase mortality. In this study clinicians were allowed to manipulate the haemodynamics according to their clinical discretion following the acquisition of data from the PAC. If specific protocols had been tested, it is possible that benefit or harm might have been demonstrated. This would be in keeping with previous studies that formally tested specific protocols. Manipulation of haemodynamics to supranormal goals in high-risk surgery has reduced mortality ${ }^{15}$ whereas similar techniques in sepsis have resulted in increased mortality ${ }^{16}$.

\section{Conclusions}

The PAC is a complex monitor that is often misunderstood. The indications for insertion of a PAC into patients are complex $^{17}$ and not based on evidence accessible to the literature. An association with an increased mortality in early trials may be due to trial design and is not backed up by prospective RCT evidence. It is more likely that any change in outcome for a patient treated with the use of a PAC is due to the therapeutic intervention rather than the PAC itself - that is, it is the clinician that causes benefit or harm, not the monitor.

\section{References}

1 Swan HJ, Ganz W, Forrester J, Marcus H, et al. Catheterization of the heart in man with use of a flow-directed balloon-tipped catheter. N Engl J Med 1970;283;447-51.

2 Mimoz O, Rauss A, Rekik N, Brun-Buisson $\mathrm{C}$, et al. Pulmonary artery catheterization in critically ill patients: a prospective analysis of outcome changes associated with catheter-prompted changes in therapy. Crit Care Med 1994;22:573-9.

3 Practice guidelines for pulmonary artery catheterization. A report by the American Society of Anesthesiologists Task Force on Pulmonary Artery Catheterization. Anesthesiology 1993;78:380-94.

4 Connors AF Jr, Castele RJ, Farhat NZ, Tomashefski JF Jr. Complications of right heart catheterization. A prospective autopsy study. Chest 1985;88:567-72.

5 Connors AF Jr, Speroff T, Dawson NV, Thomas $\mathrm{C}$, et al. The effectiveness of right heart catheterization in the initial care of critically ill patients. SUPPORT Investigators. JAMA 1996;276:889-97.

6 Dalen JE, Bone RC. Is it time to pull the pulmonary artery catheter? JAMA 1996;276 916-8.

7 Soni N. Swan song for the Swan-Ganz catheter? Br Med J 1996;313:763-4.

8 Kac G, Durain E, Amrein C, Herisson E, et al. Colonization and infection of pulmonary artery catheter in cardiac surgery patients: epidemiology and multivariate analysis of risk factors. Crit Care Med 2001;29:971-5.

9 Iberti TJ, Fischer EP, Leibowitz AB, Panacek EA, et al. Multicenter study of physicians knowledge of the pulmonary artery catheter. Pulmonary Artery Catheter Study Group. JAMA 1990;264:2928-32.

10 Iberti TJ, Daily EK, Leibowitz AB, Schecter $\mathrm{CB}$, et al. Assessment of critical care nurses' knowledge of the pulmonary artery catheter. The Pulmonary Artery Catheter Study Group. Crit Care Med 1994;22: 1674-8.

11 Trottier SJ, Taylor RW. Physicians' attitudes toward and knowledge of the pulmonary artery catheter: Society of Critical Care Medicine membership survey. New Horiz 1997;5:201-6.

12 Gore JM, Goldberg RJ, Spodick DH, Alpert JS, Dalen JE. A community-wide assessment of the use of pulmonary artery catheters in 
patients with acute myocardial infarction. Chest 1987;92:721-7.

13 Zion MM, Balkin J, Rosenmann D, Goldbourt U, et al. Use of pulmonary artery catheters in patients with acute myocardial infarction. Analysis of experience in 5,841 patients in the SPRINT Registry. SPRINT Study Group. Chest 1990;98:1331-5.

14 Rhodes A, Cusack RJ, Newman PJ, Grounds RM, Bennett ED. A randomised controlled trial of the pulmonary artery catheter in critically ill patients. Intensive Care Med (in press).

15 Boyd O, Grounds RM, Bennett ED. A randomized clinical trial of the effect of deliberate perioperative increase of oxygen delivery on mortality in high-risk surgical patients. JAMA 1993;270:2699-707.

16 Hayes MA, Timmins AC, Yau EH, Palazzo $\mathrm{M}$, et al. Elevation of systemic oxygen delivery in the treatment of critically ill patients. N Engl J Med 1994;330:1717-22.

17 Rapoport J, Teres D, Steingrub J, Higgins T, et al. Patient characteristics and ICU organizational factors that influence frequency of pulmonary artery catheterization. JAMA 2000;283:2559-67.

\section{The acute respiratory}

distress syndrome:

recent advances in

\section{ventilation}

Darryl Johnston FRCA,

Specialist Registrar in Anaesthesia

Stephen Brett MD FRCA,

Consultant in Intensive Care Medicine

Department of Anaesthesia and Intensive

Care Medicine, Hammersmith Hospital,

London

Clin Med JRCPL 2002;2:104-8

\section{Definition of the acute respiratory distress syndrome}

The acute respiratory distress syndrome (ARDS) is a diffuse pulmonary parenchymal injury associated with non-cardiogenic pulmonary oedema, resulting in severe respiratory distress and hypoxic respiratory failure. It is characterised by a triad of refractory hypoxaemia, diffuse lung infiltrates on chest radiograph and poor lung compliance. ARDS was described by Ashbaugh and colleagues in $1967^{1}$ and in subsequent studies, the diagnosis being made on clinical grounds. The current accepted definition was set out by an American-European Consensus Conference in $1994^{2}$. This defined two levels of severity in acute lung injury, based on a hypoxaemia score, ARDS being the extreme end of a spectrum of generic acute lung injury:

- acute lung injury:

$\mathrm{PaO}_{2} / \mathrm{FiO}_{2}<40 \mathrm{kPa}$;

ARDS:

$\mathrm{PaO}_{2} / \mathrm{FiO}_{2}<27 \mathrm{kPa}$

associated with:

- bilateral infiltrates on chest radiograph

- absence of cardiac failure

- acute onset of an appropriate condition.

ARDS is now regarded as the pulmonary manifestation of a systemic inflammatory response and can result from either a local (aspiration, pneumonia, smoke inhalation) or systemic insult (sepsis, trauma, pancreatitis, intravascular coagulation). Surprisingly, 95\% of deaths from ARDS are not due to hypoxaemia but to sepsis or multi-organ failure ${ }^{3}$. The prognosis is variable and depends on the underlying diagnosis, sepsis having a higher mortality. Recent studies from referral centres report an overall mortality rate of around $40 \%{ }^{4,5}$.

\section{Pathology}

The characteristic pathology of ARDS is of diffuse alveolar damage resulting in loss of integrity of the capillary-alveolar barrier, with increasing permeability and subsequent proteinaceous oedema. Surfactant dysfunction occurs, leading to loss of alveolar stability and atelectasis. This results in intrapulmonary shunting and refractory hypoxaemia ${ }^{6}$.

\section{GLOSSARY OF TERMS}

PEEP: $\quad$ positive end expiratory pressure - a ventilator mechanism which prevents alveolar pressure declining to atmospheric pressure at end expiration. It increases functional residual capacity and can maintain alveolar opening and stability.

APACHE II: acute physiology and chronic health evaluation score - a regression-derived scoring system used as an index of illness severity calculated on day one of intensive care unit stay. It produces a risk of death, but this must be used with extreme caution and not for individual patient management.

$\mathrm{FiO}_{2}: \quad$ the inspired oxygen concentration, expressed as a fraction or a percentage.

$\mathrm{PaO}_{2}$ : the partial pressure of oxygen in arterial blood, expressed as kiloPascals $(\mathrm{kPa})$ or $\mathrm{mmHg}$. 